\title{
Editorial
}

\section{International Classification of Headache Disorders - ICHD-4. Will medication-overuse headache survive?}

\author{
Mario Fernando Prieto Peres ${ }^{1}$ (D) , Thaiza Agostini Córdoba de Lima1 ${ }^{1}$ (D) , Marcelo Moraes Valença $a^{2,3}$ iD \\ ${ }^{1}$ Instituto de Psiquiatria, HCFMUSP, São Paulo, Brasil \\ Unidade de Neurologia e Neurocirurgia, Universidade Federal de Pernambuco, Recife, Brasil \\ 3Unimed Recife, Recife, Brasil
}

Mario Fernando Prieto Peres mariop3r3s@gmail.com
The fourth edition of the International Classification of Headache Disorders (ICHD-4) initiatives has been already started. A new Classification Committee leadership with Dr Peter Goadsby (chair) and Dr Stefan Evers (secretary) took over Professor Jes Olesen, who has made an incredible effort leading the first three editions. ${ }^{1.4}$

The current leaders published a letter asking for comments, suggestion for changes or improvements on the ICHD-3. ${ }^{5}$ It has already been suggested two interim modifications; first, in Definition of Terms, episodic migraine was included, defining it as a headache frequency of fewer than 15 days a month over the last 3 months, which on some days is migraine. The second, premonitory symptoms definition was corrected.

Although the extensive work and advances in the field were achieved with the first three editions, its current status is still far from ideal.

Some issues are more urgent than others. The main weakness, in our opinion, is the criteria for medication-overuse headache $(\mathrm{MOH})$ (Figure 1). The current description states that $\mathrm{MOH}$ develops "as a consequence" of regular overuse of acute medication; however, the criteria do not require any aspect of the causal relationship with acute medication use and the development of a chronic headache disorder.

Medication-overuse headache criteria do not include the definition of overuse; this is a significant drawback, by the current criteria; acute medication use, misuse, overuse, or abuse may be inadequately included, although each has a completely different meaning.

It is unsustainable for such a diagnosis without an operational definition of its main aspect: the word "overuse". The criteria in item B on 8.2.1 to 8.2.8 describes "regular intake" for " $\geq 15$ days/month for $>3$ months" in 8.2.3 and $\geq 10$ for all the others. Therefore, what the current $\mathrm{MOH}$ diagnostic criteria is diagnosing is nothing but "frequent analgesic use". Not only the lack of operational definition of overuse causes $\mathrm{MOH}$ to be ill-defined; even more confusion is brought when reading the third paragraph of the Comment section:

"The behaviour of some patients with 8.2 Medication-overuse headache is similar to that seen with other drug addictions, and the Severity of Dependence Scale (SDS) score is a significant predictor of medication overuse among headache patients." 


\section{ICHD-3 Diagnostic criteria for $8.2 \mathrm{M}$ edication-Overuse Headache}

\section{Description}

Headache occurring on 15 or more days/month in a patient with a pre-existing primary headache and developing as a consequence of regular overuse of acute or symptomatic headache medication (on 10 or more or 15 or more days/month, depending on the medication) for more than 3 months. It usually, but not invariably, resolves after the overuse is stopped.

\section{Diagnostic criteria}

A. Headache occurring on $\geq 15$ days/month in a patient with a pre-existing headache disorder

B. Regular overuse for $>3$ months of one or more drugs that can be taken for acute and/or symptomatic treatment of headache ${ }^{1 ; 2 ; 3}$

C. Not better accounted for by another ICHD-3 diagnosis.

\section{Notes}

1. Patients should be coded for one or more subtypes of 8.2 Medication-overuse headache according to the specific medication(s) overused and the criteria for each below. For example, a patient who fulfils the criteria for 8.2 .2 Triptan-overuse headache and the criteria for one of the subforms of 8.2 .3 Non-opioid analgesic-overuse headache should receive both these codes. The exception occurs when patients overuse combination-analgesic medications, who are coded 8.2.5 Combination-analgesicoveruse headache and not according to each constituent of the combination-analgesic medication.

2. Patients who use multiple drugs for acute or symptomatic treatment of headache may do so in a manner that constitutes overuse even though no individual drug or class of drug is overused; such patients should be coded 8.2.6 Medication-overuse headache attributed to multiple drug classes not individually overused.

3. Patients who are clearly overusing multiple drugs for acute or symptomatic treatment of headache but cannot give an adequate account of their names and/or quantities are coded 8.2.7 Medication-overuse headache attributed to unspecified or unverified overuse of multiple drug classes until better information is available. In almost all cases, this necessitates diary follow-up.

Figure 1. ICHD-3 Diagnostic criteria for 8.2 Medication-Overuse Headache. 
This paragraph is misleading in directing the physician in understanding overuse as abuse, addiction, or dependence. If $\mathrm{MOH}$ is then abuse, or addiction, or dependence, the specific criteria should be included. The trend in psychiatric diagnosis for substance abuse disorder in the DSM-V is to merge dependence and abuse into one set of criteria with 11 aspects6, which are not fully applicable in the reality of headache disorders and acute medication relief. The Severity of Dependence Scale suggested by the comments' paragraph also has limited applicability.

The next paragraph in the comments states that the number of days of medication use was defined based on expert opinion and not evidence. This is unacceptable in a field already significantly advanced in its scientific basis, no more expert opinions should be the basis of diagnostic criteria from now on.

"In the criteria below for the various subtypes, the specified numbers of days of medication use considered to constitute overuse are based on expert opinion rather than on formal evidence."

Moreover, $\mathrm{MOH}$ criteria generate a clinical practice dilemma, they do not accept relief in a headache day when it is the 11th or 16th headache day. If a patient has daily headaches for more than 3 months, half of their headache days will not be acutely treated.

Evidence is lacking in establishing the cause-effect of acute medication use and the worsening of headaches. Also, criteria for this aspect must be defined.

\section{References}

1. Headache Classification Committee of The International Headache Society. Classification and diagnostic criteria for headache disorders, cranial neuralgias and facial pain. Headache Classification Committee of the International Headache Society. Cephalalgia 1988;8 Suppl 7:1-96

2. Headache Classification Committee of The International Headache Society. The International Classification of Headache Disorders: 2nd edition. Cephalalgia 2004;24 Suppl 1:9-160 Doi:10.1111/j.1468-2982.2003.00824.x

3. Headache Classification Committee of The International Headache Society. The International Classification of Headache Disorders, 3rd edition (beta version). Cephalalgia 2013;33(9):629-808 Doi:10.1177/0333102413485658

4. Headache Classification Committee of The International Headache Society. Headache Classification Committee of the International Headache Society (IHS) The International Classification of Headache Disorders, 3rd edition. Cephalalgia 2018;38(1):1-211 Doi:10.1177/0333102417738202

5. Goadsby PJ and Evers S. International Classification of Headache Disorders - ICHD-4 alpha. Cephalalgia 2020;40(9):887-888 Doi:10.1177/0333102420919098

6. Livne O, Shmulewitz D, Stohl M, Mannes Z, Aharonovich E and Hasin D. Agreement between DSM5 and DSM-IV measures of substance use disorders in a sample of adult substance users. Drug Alcohol Depend 2021;227:108958 Doi:10.1016/j.drugalcdep.2021.108958 